\title{
A Kind of Neither Keynesian nor Neoclassical Model (1): The Fundamental Equation
}

\author{
Ming'an Zhan', Zhan Zhan' \\ ${ }^{1}$ Yunnan University, Kunming, China \\ ${ }^{2}$ Westa College, Southwest University, Chongqing, China \\ Email: 1792481782@qq.com, blacktea1313ryan@hotmail.com
}

How to cite this paper: Zhan, M.A. and Zhan, Z. (2016) A Kind of Neither Keynesian nor Neoclassical Model (1): The Fundamental Equation. Open Access Library Journal, 3: e3207.

http://dx.doi.org/10.4236/oalib.1103207

Received: November 6, 2016

Accepted: November 26, 2016

Published: November 29, 2016

Copyright $\odot 2016$ by authors and Open Access Library Inc.

This work is licensed under the Creative Commons Attribution International

License (CC BY 4.0).

http://creativecommons.org/licenses/by/4.0/

\begin{abstract}
This paper is different from the existing macroeconomic theory. It derives the relationship between the various macroeconomic variables from the Cobb-Douglas function, and uses statistical data in United States to verify these equations. We find that the Cobb-Douglas function is obtained in the case that the marginal output is not 0 , therefore it is a function with dynamic properties. On this basis, we use the difference between the compound output and simple output to determine the incremental output of a period, and its relationship with capital, interest rate, investment and other variables. These relations not only deny the premise of Keynesian theory, but also because the proof of the investment is not equal to the incremental capital, leaving the investment, output growth, business cycle and other neoclassical theory crisis.
\end{abstract}

\section{Subject Areas}

Economics

Keywords

Cobb-Douglas Production Function, Output, Interest Rate, Investment

\section{Introduction}

If the Cobb-Douglas function $Y=A K^{\alpha} L^{\beta}$ well be as a production model in the United States, there are two important ratios in the macroeconomic statistics: one is the income of capital/labor is about $0.3 / 0.7$ in GDP, the other is the investment/consumption is about $0.17 / 0.83$ in GDP. The former ratio indicates the allocation of output on the income of capital and labor, and the later one indicates the allocation of output on the expenditure of investment and consumption. These two ratios are both charming and 
agonizing. The charming part is these two ratios might indicate a certain rule in the macroeconomic world, which is not transferrable by people's will. The agonizing part is that current macroeconomic theories cannot explain their relations.

The fundamental principle of economics tells us that all decisions in market competition are related to their marginal state. Based on this principle, people spend more income to invest will affect the marginal state of production and the corresponding ratio of capital-labor income $(\alpha / \beta)$, as well as the ratio of investment and consumption expense $\left(I_{g} / C\right)$.

\section{The Verification of Neoclassic Investment Model}

The theory introduced by Jorgenson [1], Eisner and Strotz [2] explain the decision of investment by the opportunity cost of capital, the depreciation cost of investm8ent and the price change of investment.

Assume the marginal output of capital $K$ that the firms used is $M P K$, then the profit from the unitcapital can expressed as: $\pi=M P K-C=M P K-P_{K}\left(r_{r}+\delta\right)$. In which, $C$ is the unit cost, $P_{K}$ is the price capital, $r_{r}$ is the real interest rates and $\delta$ is the depreciation rate. In that case, the decision of investment depends on the marginal revenue produced by $K$. If the total revenue is determined by Cobb-Douglas function $Y=A K^{\alpha} L^{\beta}$, then the marginal revenue of capital $K$ is:

$$
M P K=\frac{\partial Y}{\partial K}=\frac{\alpha Y}{K}
$$

In which, the capital $K$ will reach the equilibrium status when: $M P K=C=P_{K}\left(r_{r}+\delta\right)$, take $K$ in the optimum state as $K^{*}$, then $\alpha Y / K^{*}=P_{K}\left(r_{r}+\delta\right)$ :

$$
K^{*}=\frac{\alpha Y}{P_{K}\left(r_{r}+\delta\right)}, \text { or } P_{K} K^{*}=\frac{\alpha Y}{r_{r}+\delta}
$$

The equilibrium condition reflects that the capital $K^{*}$ is negatively related to the real interest rate $r_{r}$. When $K^{*}$ move from one equilibrium status to another, and $r_{r}$ decreased, the net investment $I$ would increase $\left(I=K_{t}^{*}-K_{t-1}^{*}>0\right)$.

In Equation (2), $P_{K}$ is the price of unit capital and $P_{K} K_{r}^{*}$ refers to the total value of capital. The current $(t)$ net investment $\left(I_{t}\right)$ should equal to the difference between the current total value of capital and the earlier $(t-1)$ total value of capital $\Delta\left(P_{K} K^{*}\right)$, the gross investment $\left(I_{g t}\right)$ of period $t$ equals to the sum of this difference and depreciation $\left(D_{t}\right)$ :

$$
I_{g t}=\Delta\left(P_{K} K^{*}\right)+D_{t}=I_{t}+D_{t}=\frac{\alpha_{t} Y_{t}}{r_{r t}+\delta}-\frac{\alpha_{t-1} Y_{t-1}}{r_{r t-1}+\delta}+D_{t}
$$

then

$$
\frac{I_{g t}}{Y_{t}}=\frac{I_{t}}{Y_{t}}+\frac{D_{t}}{Y_{t}}=\frac{\alpha_{t}}{r_{r t}+\delta}-\frac{\alpha_{t-1}}{r_{r t-1}+\delta} \cdot \frac{Y_{t-1}}{Y_{t}}+\frac{D_{t}}{Y_{t}}
$$

Since there are statistical data of $I_{g}, \alpha Y, r_{r}$ and $D$ in Equation (3), as long as there is the depreciation coefficient $\delta$, we can verify whether the relation between 
these variables are corresponding to the reality in the capital market. Baro [3] estimated in his study that the measurable depreciation rate of construction and equipment stock in the US was about $5 \%$ per year. According to this number, we could calculate $I_{g t} / Y_{t}$ (model value or estimated value) in Equation (3), and compare the result to $I_{g t} / Y_{t}$ based on statistical data (statistical value). Figure 1 shows that if we calculated based on data during 1971-2015 in the United Sates, the difference between model value and statistical value would be too substantial.

In Figure 1, we notice that the changing range of $I_{g}$ from the model is wide, therefore most of the time $I_{g}$ is greater than the output level $Y$, and the maximum of $I_{g}$ is 3.353 times of GDP (year 1974). $I_{g}$ sometimes is less than 0, and the minimum value is -3.643 (Year 1976). Moreover, the average value of model values of $I_{g t} / Y_{t}$ is 0.4715 during 1971-2015, but the average value of statistical values is 0.1725 . The difference reminds us that that might be the problem of depreciation coefficient $\delta$. Only when $\delta=0.33$, the average value of model values of $I_{g t} / Y_{t}$ is close to that of statistical value.

Figure 2 shows the changing trend of model and statistical value of $I_{g t} / Y_{t}$ when $\delta=0.33$. The average value of model values during $1971-2015$ is 0.1780 , which is close to the average value of statistical values 0.1725 , and the changing range of model values narrows down. However, since $\delta=0.33$, we should deduct the $33 \%$ of capital stock

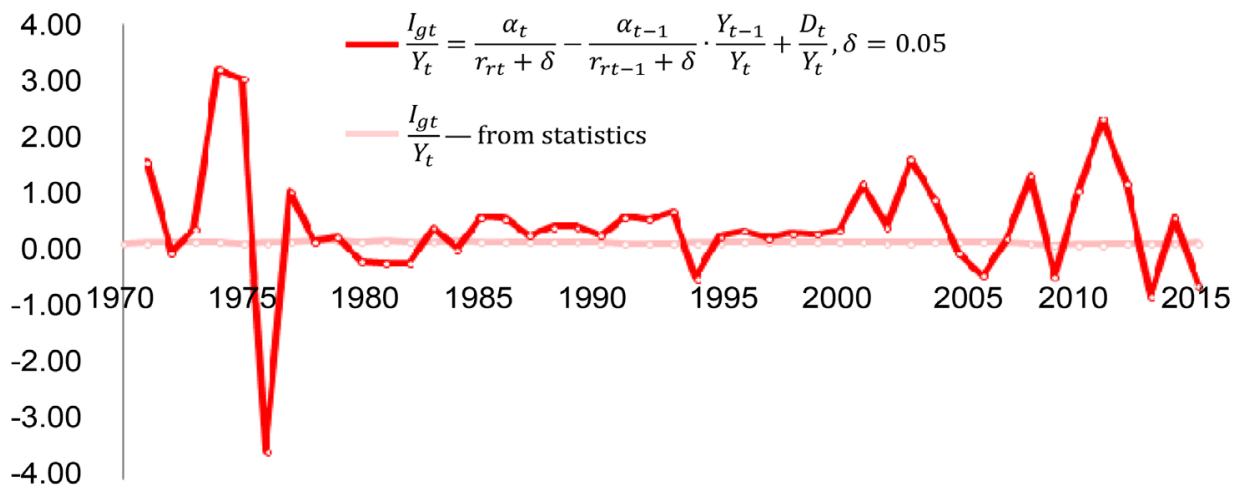

Figure 1. Model value and statistical value of $I_{g t} / Y_{t}(\delta=0.05)$. Calculation and Sources: 1) $Y_{t}=\mathrm{GDP}_{t}$. Data of GDP, $D$ and $\alpha$ are from http://www.bea.gov. $r_{r}=r-\Delta P / P$. Data of the inflation rate $\triangle P / P$ are the GDP deflator. Data of nominal interest rate $r$ are from the same as Figure 2. 2) Statistical data $I_{g t} / Y_{t}$ are calculation according to data of gross investment $I_{g}$ and GDP on http://www.bea.gov. 3) In statistical data, $Y$ not only comprises of gross investment, individual consumption, but also government expenses and net export, namely $Y=I_{g}+C+G+(X-M)$. In our calculation, we combine the government expenses $G$ in the variable $C$, and ignore the net export on the basis of: firstly, assume our analyzing object is a closed system; secondly, the value of ratio of net export and output $(X-M) / Y$ is so small that the effect of $(X-M)$ on $Y$ can be ignored. (In 1971-2015, average value of $(X-M) / Y=-0.0213$, $\left.I_{g} / Y=0.1761, C / Y=0.8451\right)$. In order to keep the equation equal, we reallocate the net export $(X-M)$ to $I_{g}$ and $C$ based on the proportion of $I_{g} / C$. Then $I_{g} / Y=0.1674, C / Y=0.8326$. 


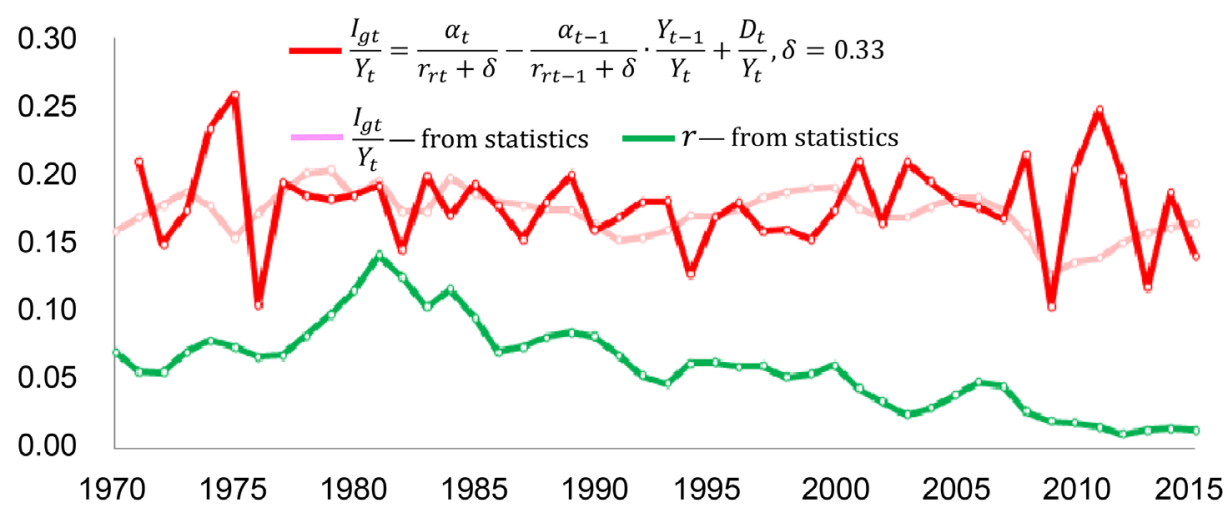

Figure 2. Model value and statistical value of $I_{g t} / Y_{t}(\delta=0.33)$. Calculation and Sources: 1) In the mode $I_{g t} / Y_{t} \quad 1, \delta=0.33$.Other data are the same as Figure 1. 2) Statistical data of interest rate $r$ are arithmetic mean of annual rates of different types of treasury constant maturities. Data of annual rates of loans are from http://www.federalreserve.gov. We use as many kinds of treasury constant maturities to calculate the average value of $r$ in order to get a value that has macroeconomic significance. Data used in this paper start in 1970 since there were enough kinds government loans since then. And value of $I_{g t} / Y$ is later than that of $r_{r t-1}$ one year, therefore the model value of $I_{g t} / Y_{t}$ starts at 1971. Correspondingly, we use data from 1971-2015. 3) Statistical values of $I_{g t} / Y_{t}$ in Figure 1 and Figure 2 are the same.

from the corresponding output. As a result, the depreciation might be greater than the output. Assume the capital stock is a times of the output: $K=a Y$, then

$D=\delta K=0.33 K=0.33 a Y$, since the output has to be greater than the depreciation:

$Y>D=0.33 a Y$, therefore $a<3.03$. Namely, the capital stock cannot be greater than 3.03 times of the output, otherwise the output would not be enough to compensate the capital depreciation. Therefore, though the average value of model values and statistical values of $I_{g t} / Y_{t}$ are generally consistent, the depreciation rate almost wears out all the output when $\delta=0.33$ and the consumption is close to 0 .

Figure 2 also shows the changing trend of model value and statistical value of $I_{g t} / Y_{t}$ are generally consistent in long-term, while they change reversely in short-term fluctuation. In order to explain the short-term change in investment, Jorgenson introduced "flexible accelerator" in the above fundamental model. Eisner and Strotz [2] added up adjusted cost in the change of investment. Lucas and Prescot [4] introduced the uncertainty of changing market. Bernanke [5], McDonald and Siegel [6], Pindyck [7] [8] discussed the irreversibility of investment with its uncertainty. It might be true that the investment would be affected by all these factors, and there might be more that affect the decision of macroeconomic investment. However, the change of interest rate has already reflected their effects. It is not that there are not enough microeconomic factors in the neoclassic investment model, but the fundamental assumption $I_{t}=\Delta\left(P_{K} K^{*}\right)$ is problematic: the net investment is not equal to the incremental capital $\Delta K$ in reality.

\section{Mysterious Fundamental Equation}

Look back on the emerging progress of Cobb-Douglas function [9]. Douglas made an 
assumption based on statistical data of United Stated during 1899-1922: in the relation between the national income $Y$, labor income $\left(Y_{L}\right)$ and capital income $\left(Y_{K}\right)$, there might be a production function. Otherwise $Y_{K} / Y$ and $Y_{L} / Y$ would not be stable at $30 \%$ and $70 \%$ about. The mathematician Charles Cobb had suggested that the capital income and labor income are determined by their marginal contribution to output $(\partial Y / \partial K, \partial Y / \partial L)$ multiply by their usage $(K, L)$, namely:

$$
Y=Y_{K}+Y_{L}=\frac{\partial Y}{\partial K} K+\frac{\partial Y}{\partial L} L
$$

Macroeconomic phenomenon that Paul Douglas observed from statistical data can be expressed as:

$$
\frac{Y_{K}}{Y}=\frac{\frac{\partial Y}{\partial K} K}{Y}=\alpha \approx 0.3, \quad \frac{Y_{L}}{Y}=\frac{\frac{\partial Y}{\partial L} L}{Y}=\beta \approx 0.7,
$$

then

$$
\frac{\partial Y}{\partial K}=\frac{\alpha Y}{K}, \quad \frac{\partial Y}{\partial L}=\frac{\beta Y}{L}, \quad \text { in which } \alpha+\beta=1
$$

The primitive function of above partial differential equations is first-degree homogeneous function $Y=A K^{\alpha} L^{\beta}$. According to the principle of general equilibrium [10], Cobb-Douglas function has possessed the character of general equilibrium: the output $Y$ that is generated by factors of production $K$ and $L$ are reallocated to these two factors based on their marginal contribution. In terms of macroeconomics, the economic profit under this condition is equal to 0 .

Cobb-Douglas function is mathematically elegant and correspondent with economic principles (diminishing marginal output). Since it is generated from the deduction of macroeconomic phenomenon, we need not to study its rationality by building a typical firm. The assumption of a "typical firm" is might be wrongful cause by "fallacy of composition". Since the increase of income of one firm might be related to the decrease of others, therefore the income of the system would not be increased as the "typical firm". Cobb-Douglas function is deduced under the assumption that $\partial Y / \partial K$ and $\partial Y / \partial L$ are not equal to 0 , therefore it is a dynamic function with macroeconomic factors. In other words, the output in Cobb-Douglas function is always changing. Now the question is, how can we get the growth of output $\Delta Y$ or the grow rate of output $\Delta Y / Y$ implied by Cobb-Douglas function?

If $Y=A K^{\alpha} L^{\beta}$ was a production function at some point of time, then the variable should has $t$ as the subscript: $Y_{t}=A_{t} K_{t}^{\alpha_{t}} L_{t}^{\beta_{t}}$. In which, $K$ and $L$ in Cobb-Douglas function might change with the changing $Y$, especially $K$. Since,

$$
M P K=\frac{\partial Y}{\partial K}=\frac{\alpha_{t} Y_{t}}{K_{t}} \text {, and } \frac{\partial Y}{\partial K} \approx \frac{\Delta Y}{\Delta K},
$$

then

$$
\Delta K \approx \frac{K_{t}}{\alpha_{t} Y_{t}} \Delta Y
$$


Equation (8) indicates that the capital stock $K$ will increase with the increasing output at some point of time. More specifically, the relation between the output $Y$ during a period and the output at a point of time $Y_{t}$ should be:

$$
Y=\int_{t_{1}}^{t_{2}} Y_{t} \mathrm{~d} t=\int_{t_{1}}^{t_{2}} A_{t} K_{t}^{\alpha_{t}} L_{t}^{\beta_{t}} \mathrm{~d} t
$$

If $K$ and $L$ stayed unchanged, then $Y$ would be equal to $\left(Y_{t_{2}}-Y_{t_{1}}\right)$. However, if the output at a point of time took part in the output of next period with $K_{t}$ and $L_{t}$, then $Y$ would be greater than the one calculated with unchanged stock $K$ and $L$. The difference is similar to that of simple and compound interest accrual in savings. Therefore we call the output that is not affected by the change of $K$ and $Y$ as the "simple output" and which is affected by the change of $K$ and $Y$ as the "compound output".

Based on the feature of Cobb-Douglas function, the marginal output of $K(M P K)$ has nothing to do with $L$. Therefore we could assume the market interest rate is equal to $M P K$ and the output $Y$ can be expresses as $\alpha Y=r K$. This equation indicates that we can get $\alpha Y$ with knowing $K$ at any point of time and marginal revenue $r$. When the current $r K$ takes part in the output of next period and $\alpha$ keeps unchanged, then the compound output $Y_{C}$ of difference times can be calculated as below.

Single compound output in a year: $\alpha Y_{C}=r K$. Divide the output of a year into two part, and calculate the compound output of half a year based on $r K / 2$, then multiply by 2 to get the compound output in this year: $\alpha Y_{C}=[r K / 2+r(r K / 2)] \times 2=r K+r^{2} K$. In a similar way, the compound output of $n$ times a year should be:

$$
\alpha Y_{C}=\left[\frac{r K}{n}+r\left(\frac{r K}{n}\right)+\cdots+r^{n-1}\left(\frac{r K}{n}\right)\right] \times n=r K+r^{2} K+\cdots+r^{n} K
$$

When $n \rightarrow \infty$, then:

$$
\alpha Y_{C}=\sum_{n=1}^{\infty} K r^{n}=r K \sum_{n=1}^{\infty} r^{n-1}, \text { in which }|r|<1,
$$

then

$$
Y_{C}=\frac{r K}{\alpha(1-r)}
$$

In the above equation, the condition that the interest rate $r$ should not be more or equal to 1 would not affect the theoretical meaning. Since if $r$ was more or equal to 1 in a certain period, as long as we shorten the period of time, then we could meet the requirement of $|r|<1$. Certainly the measuring period of $Y$ should be correspondingly shortened as well. For example, when the annual interest rate is 2, Equation (8) is still effective when we applies seasonal interest rate and seasonal output.

When the interest rate $r$ is 0 , then there is no difference between the simple and compound output, the higher of the interest rate, the greater of compound output. When $r=0.1$, the compound output is 1.11 times of simple output $(=1 / 0.9)$. The concept of "compound output" is everywhere in output activities, though people do not see them that way. According to Equation (8), the relation between the compound and simple output is $Y_{C}=Y /(1-r)$. 
We take $Y$ as the compound output, and $Y_{S}=(1-r) Y$ as the simple output. In that case, we could use the signs in Cobb-Doulas function. $Y$ and $Y_{S}$ can be expressed as:

$$
Y=\frac{r K}{\alpha}, Y_{S}=(1-r) Y=(1-r) \frac{r K}{\alpha}
$$

Equation (9) shows that when $r=0$, that $Y=Y_{S}$. Therefore there is no increase of the output, or in other words, the output at the end of period $Y_{t_{2}}$ is the same as the output at the beginning of period $Y_{t_{1}}$. From the aspect of time, the simple output $Y_{S}$ is the output at the beginning of period $Y_{t_{1}}$, and the compound output is the output at the end of period $Y_{t_{2}}$. As long as $r>0$, then $Y_{t_{2}}>Y_{t_{1}}$. Cobb-Douglas in which the marginal output is not 0 has imply the change state of output, and the change rate of output is:

$$
\frac{Y_{t_{2}}-Y_{t_{1}}}{Y_{t_{1}}}=\frac{Y-Y_{S}}{Y_{S}}=\frac{Y}{Y_{S}}-1=\frac{Y}{(1-r) Y}-1=\frac{r}{1-r}
$$

The above equation shows that the theoretical change rate of output is a function of the interest rate $r$. We need to remember that $Y$ in the denominator is $Y_{t_{1}}$ or the simple output $Y_{S}$, rather than $Y_{t_{2}}$ or the compound output $Y$. Since Equation (10) is deduced under the assumption of $r=\partial Y / \partial K$, it has to be verified by statistical data.

During 1971-2015, the average value of the growth rate of nominal GDP is 0.0651, the average value of nominal interest rates is 0.0604 during the same period, $r /(1-r)=0.0604 /(1-0.0604)=0.0643$, which is close to 0.0651. Since Equation (10) is an approximate expression without considering the periodic relation between growth rate and interest rate (after some time, we will see the difference of $\Delta Y / Y$ and $r$ in short-term fluctuation), it is can only be verified by relatively long-term statistical average values.

When $r=0$, we cannot take the simple and compound output are also equal to 0 based on Equation (9) $Y=r K / \alpha$, since the original meaning of $r=0$ is that the marginal output of $K$ is 0 and the growth rate of output is 0 . Therefore, the meaning of $r=0$ here is that the compound output is equal to the simple output, or the output at the end of period is equal to that at the beginning of period. In terms of economic meaning, the marginal output is 0 has two different meanings. One is that the total output has reached the maximum and the economy only need to simply reproduce year by year; or the economic systems has lost dynamism and can only reproduce on a low level. The simple reproduction is a state that the output is completely consumed, namely the sum of the consumption $C$ and the depreciation $D$ is equal to the total output. Therefore the net investment I can only exist when $Y>Y_{S}$ or $Y_{t_{2}}>Y_{t_{1}}$. According to Equation (9), the difference between the end-of-period and beginning-of-period output is:

$$
\Delta Y=Y-Y_{S}=Y_{t_{2}}-Y_{t_{1}}=\frac{r K}{\alpha}-\frac{r(1-r) K}{\alpha}=r \cdot \frac{r K}{\alpha}=r Y
$$

The above shows $\Delta Y$ implied in Cobb-Douglas function. Although the net investment $I$ is generated from the newly increased output $\Delta Y, \Delta Y$ also comprises of the 
newly increased consumption and depreciation, therefore $I \leq \Delta Y$. $I \leq \Delta Y$ is established when $r=0$. Since $\Delta Y=r Y$ and $Y_{t_{1}} \leq Y_{t_{2}}$, when assumed $I=r Y_{S}$, then the value of $I$ is not only in a critical state that $I=\Delta Y=0$ when $r=0$, and accords with the logical requirement of $I \leq \Delta Y$. Under the assumption $=r Y_{S}$, the relation between the net investment $I$ and $Y$ is:

$$
I=\Delta Y(1-r)=r Y(1-r), \text { or } \frac{I}{Y}=r(1-r)
$$

The above equation shows the internal relation between the net investment, output and the interest rate. We call Equation (12) the investment equation. Equation (12) is deduced by assuming $I=r Y_{t_{1}}$, the rationality of the equation need to be testified by statistical data. During 1971-2015 in the United States, the average value of statistical data of $I_{g} / Y$ and $D / Y$ are 0.1725 and 0.1169 , respectively. Therefore $I / Y=I_{g} / Y-D / Y=0.1725-0.1169=0.0556$. During the same period, the average value of $r$ is 0.0604 , and the value of $I / Y$ which is estimated according to $r$ is $I / Y=r(1-r)=0.0604(1-0.0604)=0.0567$. The statistical value 0.0556 is close to the estimated value 0.0567 .

From the aspect of microeconomics, on one hand the lower of interest rate, the greater of firm investment under certain output. But when all firms increase their investments, then the interest rate will increase; on the other hand, the cut of consumption will increase savings in households. But when the economy is under a low level of interest rate, some people would found to consume others' savings on mortgage will increase their benefit level. Therefore it will lead to the increase of interest rate. As a result, if the interest rate was determined by the market competition, the investment or savings is not necessarily negatively-related to the interest rate. The macroeconomic investment equation reflects this relation and is not contradictory to the microeconomic formation of investment.

\section{Verification and Deduction of the Fundamental Equation}

Equation (12) is the net investment equation. In order to compare with the short-term changing tendency of the gross investment $I_{g}$ in statistical data, we need to expand Equation (12) to the gross investment equation:

$$
\frac{I_{g}}{Y}=\frac{I}{Y}+\frac{D}{Y}=r(1-r)+\frac{D}{Y}
$$

As Figure 3 shows, the fluctuation of $D / Y$ is narrower than that of the interest rate $r$, and the fluctuation of $I_{g} / Y$ is determined by that of $I / Y$. In that case, we consider the theoretical estimation of $I_{g} / Y$ equal to the estimation value of $I / Y=r(1-r)$ plus the statistical value of $D / Y$.

Figure 3 shows that $I_{g} / Y$ based on Equation (13) is much better than that in neoclassic investment model. However, compared to the statistical value, the shortterm fluctuation range are slightly different. The main reason is that Equation (13) is deduced on the assumption $\alpha$ and $r$ are constants, or in other words, Equation (13) 


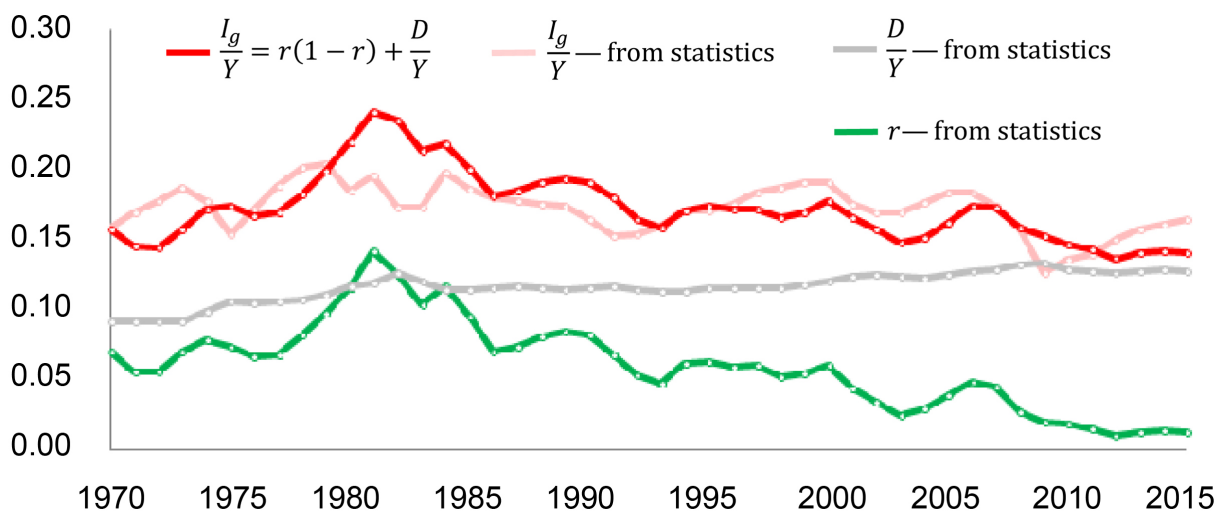

Figure 3. Model value and statistical value of $I_{g} / Y$. Calculation and Sources: Statistical values of $D / Y$ and $I_{g} / Y$ are the same as Figure 1 . In the model $I_{g} / Y=r(1-r)+D / Y$, nominal interest rates $r$ are from the same as Figure 2.

neglects the effect of $\Delta \alpha$ and $\Delta r$ on $I / Y$.

In our later study of business cycle, we will learn that although in long term that $\Delta Y / Y=r$ is established, there is always phase difference of $\Delta Y / Y$ and $r$ in short term. Therefore, if we use $\Delta Y / Y$ to replace $r$ in the investment equation, then the model value of $I / Y$ or $I_{g} / Y$ would also change correspondingly. According to Equation (11), substitute $r=\Delta Y / Y$ to Equations (12) or (13):

$$
\frac{I}{Y}=\frac{\Delta Y}{Y}\left(1-\frac{\Delta Y}{Y}\right), \text { or } \frac{I_{g}}{Y}=\frac{\Delta Y}{Y}\left(1-\frac{\Delta Y}{Y}\right)+\frac{D}{Y}
$$

The average value of $I_{g} / Y$ of the United States during 1971-2015 is 0.1768 (calculated values based on Equation (13) is $r(1-r)+D / Y=0.0567+0.1169=0.1736$ ). And the short-term fluctuation is as Figure 4 shows. Compared to Figure 3, the fluctuation of $I_{g} / Y$ is more consistent with statistical values in short term, while different when the inflation rate is comparatively high or low. The reason is that the inflation rate $\Delta P / P$ is greater or smaller than the real interest rate $r_{r}$ or the growth rate of real output $\Delta Y_{r} / Y_{r}$. We will elaborate on this in the analysis of inflation.

In the deduction of the difference of $\Delta Y=r Y$ which is the difference of $Y_{t_{2}}$ and $Y_{t_{1}}$, we assume $\alpha$ and $r$ are exogenous variables. And if we use the same assumption in Equation (9) $Y=r K / \alpha$, then $\Delta K=(\alpha / r) \Delta Y$. Since $\Delta Y=r Y$, therefore:

$$
\Delta K=\alpha Y
$$

$\Delta K$ in Equation (15) not only shows the relation between the growth of $K$ and the output $Y$, but also reflects the share of capital revenue in the output. For better understanding of this relation under the state of simple reproduction and that of extended reproduction, we create Figure 5 to show the relation of allocation of output in terms two incomes and two expenses. In reality, the output move from a state of extended reproduction to another state of extended reproduction, therefore the change of $\alpha$ is quite small and would not be as great as the following figure shows.

Figure 5 shows that if $D / Y$ is a constant, then $I / Y$ (or $C / Y$ ) is closely related to 


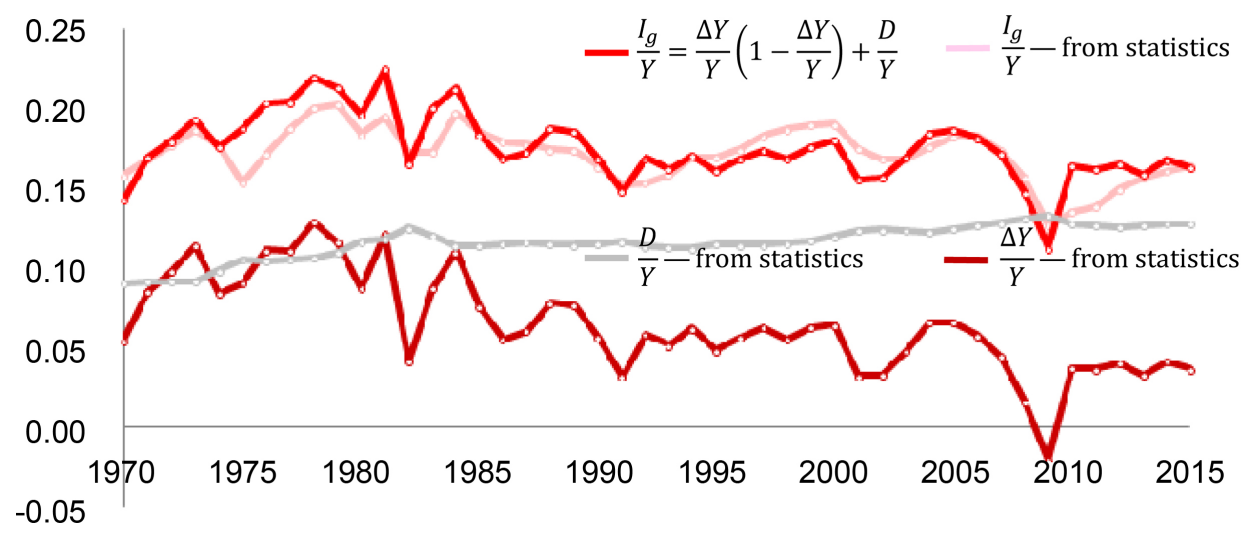

Figure 4. Model and statistical values of $I_{g} / Y$. Calculation and Sources: Statistical values of $I_{g} / Y$ and $D / Y$ are the same as Figure 3. In the model $I_{g} / Y=\Delta Y / Y(1-\Delta Y / Y)+D / Y$, nominal interest rate are the same as Figure 2, $\Delta Y / Y$ is the growth rate of nominal GDP, data are from http://www.bea.gov/.

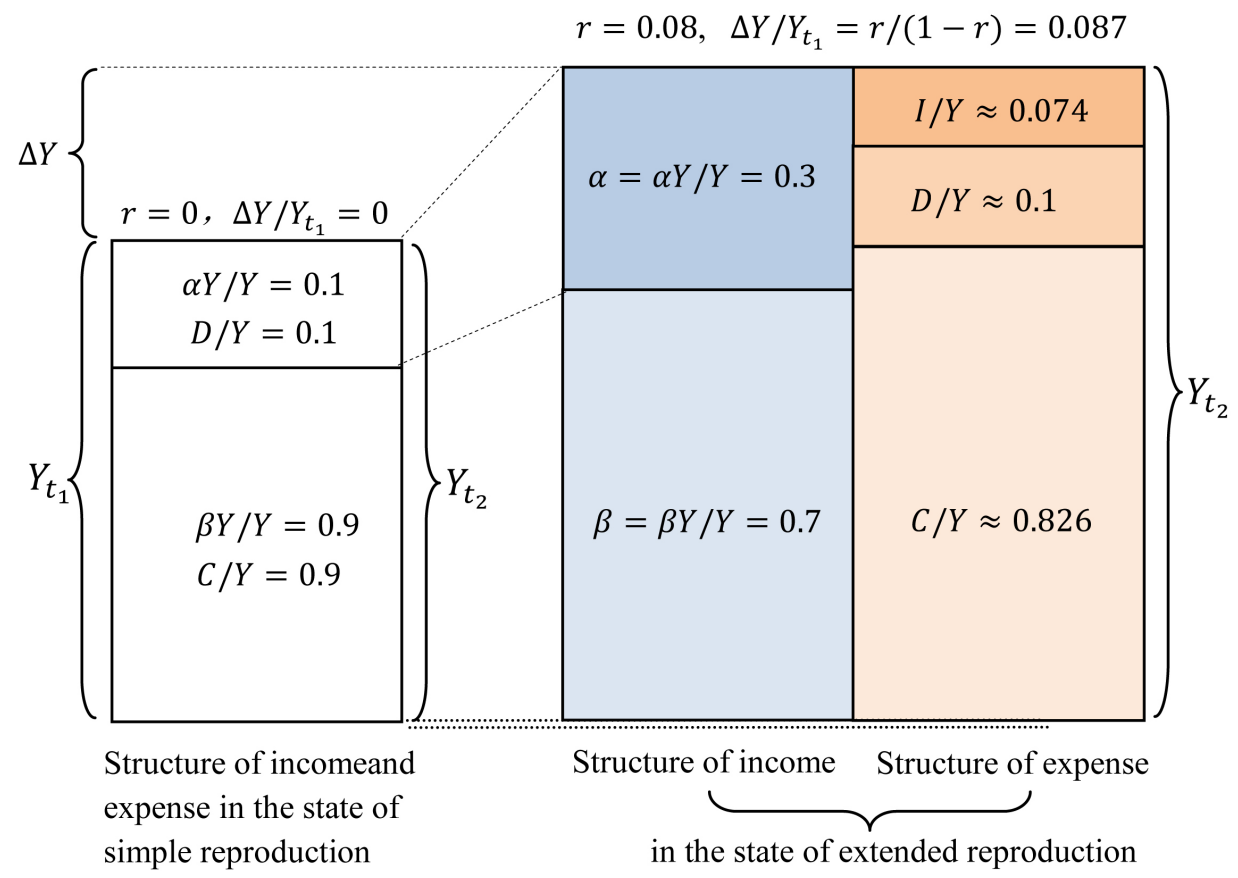

Figure 5. Difference of structure of expense and income in the simple reproduction and the extended reproduction. Notice: 1$)$. In simple reproduction, $r=0$, therefore $I / Y=r(1-r)=0$; in the growing output, assume $r=0.08$, therefore $I / Y=0.08(1-0.08)=0.074$. Assuming $D / Y=0.1$, then $C / Y=1-I / Y-D / Y=0.826 .2)$. Assume $D / Y$ are the same in the state of simple reproduction and growing output. Other data are examples with different assuming data.

the marginal output of capital $r$. When $r=0$, the capital return $\alpha Y$ would be used up by depreciation. And only when $r>0$, there would be net investment $I$. Though the greater value of $r$, the less value of $C / Y$, the total consumption $C$ always increases when $r>0$. It indicates that $r$ determines the distribution of the net investment and 
assumption while determines the growth rate of output.

Based on $\Delta Y=r Y$, not only we can deduce $\Delta K=\alpha Y$, but also other important inference. According to Equation (9), $K=\alpha Y / r$, we can get

$$
\frac{\Delta K}{K}=\frac{\alpha Y}{\alpha Y / r}=r \text {, or } \Delta K=r K
$$

Assume the current gross capital equals to the sum of the beginning-of-period capital and the growth of capital $K_{t_{2}}=K_{t_{1}}+\Delta K$ or $K=K_{S}+\Delta K$. Since $K=\alpha Y / r$ and $\Delta K=\alpha Y$, then:

$$
K_{t_{1}}=K-\Delta K=\frac{\alpha Y}{r}-\alpha Y=\left(\frac{1}{r}-1\right) \alpha Y
$$

According to $I=r(1-r) Y$ and $\Delta K=\alpha Y$, then:

$$
\Delta K=\frac{\alpha}{r(1-r)} I
$$

Since $\alpha \gg r$, therefore when $r>0$,

$$
\Delta K=\frac{\alpha}{r(1-r)} I>\frac{\alpha}{r} I \gg I
$$

Based on the average value of $\alpha / r=0.3251 / 0.0604=5.382$ during 1971-2015, $\Delta K>5.382 I$. The neoclassic investment model which is deduced as assuming $I=\Delta K$ cannot be verified by statistical data. According to the hypothesis of $r=\partial Y / \partial K=\alpha Y / K$, if $r$ and $\alpha$ don't change with $Y$ and $K$, then $\Delta K=(\alpha / r) \Delta Y$. Even if incremental output be used all to investment, namely $\Delta Y=I$, we will also get a conclusion: $\Delta K \gg I$, because $r \ll \alpha$.

Since $\Delta K \gg I$, if we assume $I=\Delta K$, the estimates value of $I_{g} / Y$ based on Equation (2) when $\delta=0.05$ would be much greater than the statistical value. In Figure 2, in order to reduce the substantial difference of the estimates value according to neoclassic model and the statistical data, we have to set the depreciation coefficient $\delta$ in the model as 0.33 .

In our model, the net investment $I$ and the incremental capital $\Delta K$ are both deduced by the difference between the compound and simple output $\Delta Y$. Since $I$ is part of $\Delta Y$ (because $I=(1-r) r Y<r Y=\Delta Y$ ), and $\Delta K$ is much greater than $\Delta Y$ (because $\Delta K=\alpha \Delta Y / r>\Delta Y$ ), the capital growth much be greater than the net investment. However, this conclusion is very confusing in reality, since "capital origins from investment" has been accepted as a "truth" for economist and the public. If $\Delta K>I$, there where the difference comes from?

By taking differential of both sides of $K=K_{S}+\Delta K$, we can get $\Delta K=\Delta K_{S}+\Delta \Delta K$, in which $K_{S}$ is the capital stock of the current period. It indicates that the value and stock of $\Delta K$ are related to the growth of the capital stock $K_{S}$. In a state where the compound output $Y$ is greater than the simple output $Y_{\mathcal{S}}$ there must be $\partial Y / \partial K=r>0$, in which $\partial K$ refers to $\Delta K$. When $\Delta Y>0$, all capitals including the capital stock would increase. We also need to remember that the increase of capital stock is not nec- 
essarily caused by the increase of quantity of equipments, it can also be caused by improving the operating efficient of old equipments.

Though we cannot directly get $\Delta K_{S}$ from statistical data, if $r>0$ in the current period, we can get $\Delta K$ and corresponding $\Delta K_{S}$ based on $r=\alpha Y / K$ and $\Delta K=\alpha \Delta Y / r$. Since, $K=K_{S}+\Delta K, \Delta K=\Delta K_{S}+\Delta \Delta K, \Delta K=\alpha Y$, If $\alpha$ was a constant, then, $\Delta K_{S}=\Delta K-\Delta \Delta K=\alpha Y-\Delta(\alpha Y)=\alpha Y-\alpha \Delta Y=\alpha(Y-\Delta Y)=\alpha Y_{S}$, in which $Y_{S}=(1-r) Y$, then $\Delta K_{S}=\alpha(1-r) Y>I=r(1-r) Y$ (in which $\alpha>r$ ).

The above equation indicates that the capital grow is not restricted to the visible growth of investment. In the study of economic growth, some people emphasized the impact of human resources, others emphasize that of economic system, etc.. In fact, all these impact are working at the same time. The incremental capital $\Delta K_{S}$ is the consequence of all impacts. Also in reality, the improvement of employees' qualities could maximize the capability of old equipments. This can be categorized as the impact of human resources. A typical example of the economic system put impact on the output is those countries transfer from planned economies to market economies. Although these countries have very high ratio of investment-output before their economic reformation, they could not get corresponding growth rate of output. And after their economic reformation, the same capital stock can produce more products.

The investment $I$ is a special variable. In the identical equation $Y=I+C+D, I$ is a total value. However, in the dynamic compound output, $I$ is part of the incremental output $\Delta Y$, and $I / Y \leq \Delta Y / Y$, or $I_{r} / Y_{r} \leq \Delta Y_{r} / Y_{r} \quad\left(I_{r}\right.$ and $Y_{r}$ is the real investment and output). We cannot assume $Y=P Y_{r}$ and $I=P I_{r}$ at the same time ( $P$ is the price index), otherwise there would the contradiction that $I / Y=\left(P I_{r}\right) /\left(P Y_{r}\right)=I_{r} / Y_{r}$. As a result, when we assume $Y=P Y_{r}, I / Y$ should be considered as an integral whole. Based on $(I / Y)=\Delta Y / Y(1-\Delta Y / Y)$, we define the $(I / Y)_{r}$ as:

$$
\left(\frac{I}{Y}\right)_{r}=\frac{\Delta Y_{r}}{Y_{r}}\left(1-\frac{\Delta Y_{r}}{Y_{r}}\right)
$$

then

$$
\left(\frac{I}{Y}\right)-\left(\frac{I}{Y}\right)_{r}=\frac{\Delta Y}{Y}\left(1-\frac{\Delta Y}{Y}\right)-\frac{\Delta Y_{r}}{Y_{r}}\left(1-\frac{\Delta Y_{r}}{Y_{r}}\right)=\frac{\Delta P}{P}\left[1-\left(\frac{\Delta Y}{Y}+\frac{\Delta Y_{r}}{Y_{r}}\right)\right] \geq 0
$$

In which, $Y=P Y_{r}, \Delta P>0$, and assume $\left(\Delta Y / Y+\Delta Y_{r} / Y_{r}\right)<1$. Since $\Delta P / P>0$ during 1971-2015, therefore $(I / Y)>(I / Y)_{r}$ as Figure 6 shows. Though $(I / Y) \neq(I / Y)_{r}$, the fluctuation of $(I / Y)$ and $(I / Y)_{r}$ is generally consistent in short term, which indicates that the shape of fluctuation of $(I / Y)$ and $\left(I_{g} / Y\right)$ is determined by the change of $\Delta Y_{r} / Y_{r}$.

Since $I$ is part of the growth of output, the growth rate of investment $\Delta I / I$ is a higher order variable compared to the growth rate of output, therefore the changing pattern of $\Delta I / I$ might not be the same as that of $I / Y$. According to the identical equation $\Delta(I / Y) /(I / Y)=\Delta I / I-\Delta Y / Y$, then:

$$
\frac{\Delta I}{I}=\frac{\Delta Y}{Y}+\frac{\Delta(I / Y)}{I / Y} \text {, and } \frac{I}{Y}=\frac{\Delta Y}{Y}\left(1-\frac{\Delta Y}{Y}\right),
$$




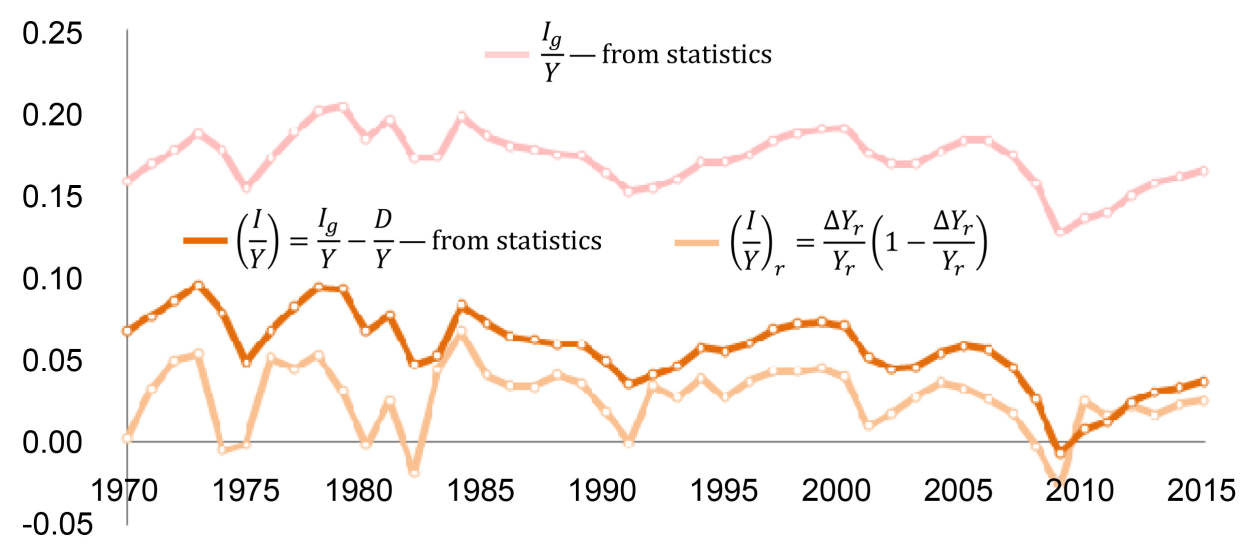

Figure 6. Compare between $(I / Y)_{r}$ and $(I / Y)$. Calculation and Sources: In equation $(I / Y)_{r}=\Delta Y_{r} / Y_{r}\left(1-\Delta Y_{r} / Y_{r}\right), \Delta Y_{r} / Y_{r}=\Delta Y / Y-\Delta P / P$, data of $\Delta P / P$ see Figure 1. Data of $I_{g} / Y$ and $D / Y$ are the same as Figure 1.

then

$$
\frac{\Delta I}{I}=\frac{\Delta Y}{Y}+\frac{1-2(\Delta Y / Y)}{1-(\Delta Y / Y)} \cdot \frac{\Delta(\Delta Y / Y)}{(\Delta Y / Y)} \approx \frac{\Delta Y}{Y}+\frac{\Delta(\Delta Y / Y)}{(\Delta Y / Y)}
$$

Since the change of output is the reason of the change of investment, then the ratio of consumption and output is determined by the economical system at the same time when it determined the ratio of investment and output. According to the statistical identical equation $Y=C+I_{g}=C+I+D$, then:

$$
\frac{C}{Y}=1-\frac{I_{g}}{Y}=1-\left[\frac{\Delta Y}{Y}\left(1-\frac{\Delta Y}{Y}\right)+\frac{D}{Y}\right]
$$

Because of the fluctuation range of $D / Y$ is quite small compared to $\Delta Y / Y$, we assume $D / Y$ is a constant. Therefore, we can deduce $C / Y$ would decrease when $\Delta Y / Y$ increased according to Equation (20). Since $C / Y$ and $\Delta C / C$ are two different variables, therefore when $\Delta Y / Y$ increases, $\Delta C / C$ would not necessarily decrease. According to $\Delta(C / Y) /(C / Y)=\Delta C / C-\Delta Y / Y$, then:

$$
\frac{\Delta C}{C}=\frac{\Delta Y}{Y}+\frac{\Delta\left(1-I_{g} / Y\right)}{1-I_{g} / Y}
$$

In which

$$
\begin{gathered}
\frac{\Delta\left(1-I_{g} / Y\right)}{1-I_{g} / Y}=-\frac{I / Y}{\left(1-I_{g} / Y\right)} \cdot \frac{\Delta(I / Y)}{(I / Y)}=-\frac{I / Y}{\left(1-I_{g} / Y\right)}\left(\frac{\Delta I}{I}-\frac{\Delta Y}{Y}\right), \quad \text { According to E. } \\
\frac{\Delta I}{I} \approx \frac{\Delta Y}{Y}+\frac{\Delta(\Delta Y / Y)}{(\Delta Y / Y)}
\end{gathered}
$$

then

$$
\frac{\Delta C}{C}=\frac{\Delta Y}{Y}-\frac{I / Y}{\left(1-I_{g} / Y\right)}\left(\frac{\Delta I}{I}-\frac{\Delta Y}{Y}\right) \approx \frac{\Delta Y}{Y}-\frac{I}{C} \cdot \frac{\Delta(\Delta Y / Y)}{(\Delta Y / Y)}
$$




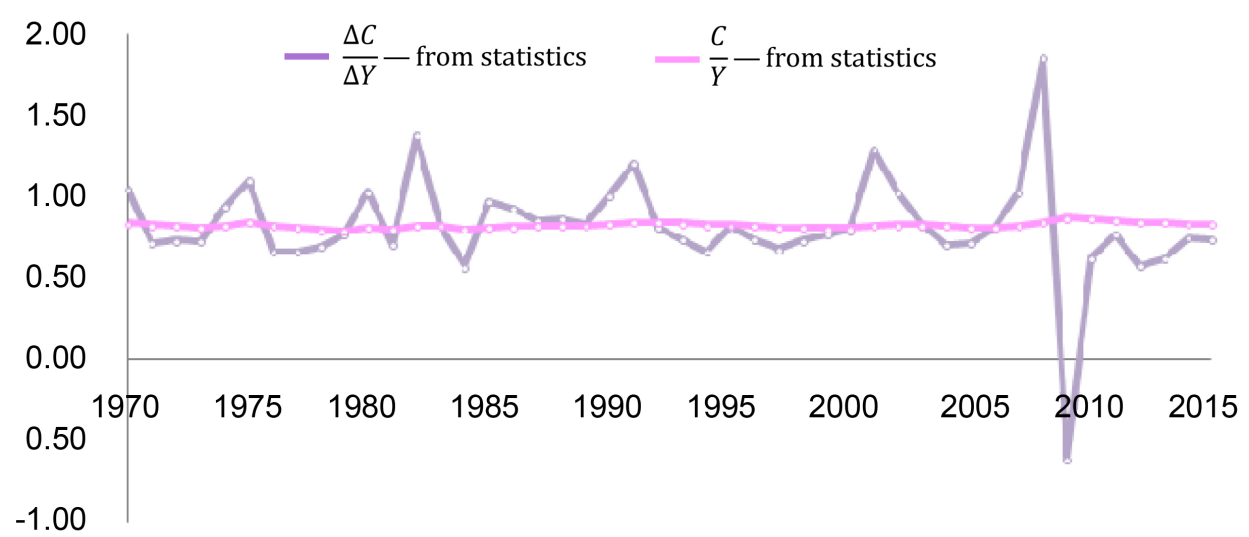

Figure 7. Fluctuates in short term and trends in long term of $\Delta C / \Delta Y$ and $C / Y$. Calculation and Sources: Data of $C$ and $Y$ are from the same as Figure.

In Equation (21), $\Delta C / C$ is mainly affected by $\Delta Y / Y$. However, since the secondorder growth rate $\Delta(\Delta Y / Y) /(\Delta Y / Y)$ has opposite effects on $\Delta C / C$, therefore the shape of fluctuation of $\Delta C / C$ and $\Delta Y / Y$ might be different.

We can also deduce the theoretical expression of the marginal propensity of assumption $\Delta C / \Delta Y$ based on Equation (21), and deny the assumption of diminishing marginal propensity of Keynes [11]. According to $(\Delta C / C) /(\Delta Y / Y)=(\Delta C / \Delta Y) /(C / Y)$, then:

$$
\frac{\Delta C}{\Delta Y}=\frac{C}{Y} \cdot \frac{\Delta C / C}{\Delta Y / Y}=\frac{C}{Y} \cdot \frac{\Delta Y / Y-(I / C) \frac{\Delta(\Delta Y / Y)}{(\Delta Y / Y)}}{\Delta Y / Y}=\frac{C}{Y}\left(1-\frac{I}{C(\Delta Y / Y)} \cdot \frac{\Delta(\Delta Y / Y)}{(\Delta Y / Y)}\right)
$$

In Equation (22), $\Delta Y / Y$ fluctuates in short term, and the second-order growth rate $\Delta(\Delta Y / Y) /(\Delta Y / Y)$ is either more than 0 or less than 0 , therefore the marginal propensity of assumption $\Delta C / \Delta Y$ is possibly greater or less than the average propensity of assumption $C / Y \quad(\Delta C / \Delta Y \gtrless C / Y)$.

In long term, if the average value of the second-order growth rate $\Delta(\Delta Y / Y) /(\Delta Y / Y)$ is equal to 0 , then $\Delta C / \Delta Y=C / Y$; if the output growth rate is decreasing, namely $\Delta(\Delta Y / Y) /(\Delta Y / Y)<0$, then $\Delta C / \Delta Y>C / Y$. As Figure $7, \Delta C / \Delta Y$ is fluctuation around $C / Y$ in the short term, and is not decreasing trend obviously. Both of the average value are 0.8171 and 0.8275 during 1971-2015. Therefore, Keynes's assumption of consumption is not certificated in our theory and statistical data.

\section{Conclusions}

\subsection{Hypothesis}

$\diamond$ Production function in the market system: $Y=A K^{\alpha} L^{\beta}$.

$\diamond$ A marginal condition: $r=\partial Y / \partial K=\alpha Y / K$.

$\diamond$ According to the compound interest method, calculate the output at the end of pe$\operatorname{riod} Y_{t_{2}}: Y_{t_{2}}=Y=Y_{t_{1}} /(1-r)$. 
Net investment: $I=r Y_{t_{1}}$.

\subsection{Results}

$\diamond$ Fundamental equation: $\Delta Y=r Y, \Delta K=r K$.

$\diamond$ Investment equation: $I / Y=r(1-r)$ or $I / Y=(\Delta Y / Y)(1-\Delta Y / Y)$.

$\diamond$ The incremental capital is not equal to the investment: $\Delta K=[\alpha / r(1-r)] I$.

\subsection{Discussion}

$\diamond$ In the analysis method, this paper seems to eliminate the gap between the traditional macroeconomics and the microeconomics. Cobb-Douglas function used in this paper not only have the property of diminishing marginal returns, but also be constrained by marginal conditions $r=\partial Y / \partial K$.

$\diamond$ For statistical identity $Y=C+I_{g}+(X-M)$, many people believe that the right side (demand) of what factors determines the left side (supply). The reasoning process of this paper shows that the marginal state of capital determines the growth of output, which determines the investment and also determines the distribution of investment and consumption in the total output, because the left side of the identity is determined by $Y=A K^{\alpha} L^{\beta}$ in this paper, and is not determined by investment and consumption.

$\diamond$ The result of this paper shows $\Delta K \neq I$, but from the savings model of Ramsey [12], the output growth model of Solow [13], the investment model of Jorgenson [1] to the real business cycle model of Kydland and Prescott [14] [15], all of these apply the equation of $\Delta K=I$ to analyze macroeconomic problems. If $\Delta K=[\alpha / r(1-r)] I$ is substituted for equation $\Delta K=I$, the target functional in DSGE (Dynamic Stochastic General Equilibrium) will not converge. This means that we need to re-examine those neoclassic theory that use dynamic optimization as a reasoning tool.

$\diamond$ We derive the relationship between output, interest rate, investment and other variables from Cobb-Douglas function and the corresponding marginal conditions, but some of the variables in the equation can only be verified with the long-term average of the statistics (such as $\Delta Y=r Y$ ). This may be related to the periodicity of variations in the short-term. In addition, we assume that $r$ is constant when calculating the incremental output $\Delta Y$, since if $r$ was variable that the rate of output change would depend not only on $r$ but also on the rate of change of $r$. The equations in this paper are only the basis for our future analysis of more problems. To relax some of these assumptions, we can further analyze the macroeconomic issues such as the economic cycle, inflation and economic growth, and finally form a logical and complete theoretical system.

$\diamond$ At present, these equations of the paper are not suitable for the analysis of nonmarket system (for example, the market interest rate is limited by the government in China). In addition, another shortcoming of these models is that they are not used to validate more market system countries, since many countries are not as open and complete as the United States. 


\section{References}

[1] Jorgenson, D. (1963) Capital Theory and Investment Behavior. American Economic Review, 53, 247-259.

[2] Eisner, R. and Strotz, R.H. (1963) Determinants of Business Investment, in Commission on Money and Credit, Impacts of Monetary Policy. Prentice-Hall, New Jersey, 59-337.

[3] Barro, R.J. and Sala-I-Martin, X. (1995) Economic Growth. McGraw-Hill, New York.

[4] Lucas, R.E.J. and Prescott, E.C. (1971) Investment under Uncertainty. Econometrica, 39, 659-681. https://doi.org/10.2307/1909571

[5] Bernanke, B.S. (1983) Irreversibility, Uncertainty and Cyclical Investment. Quarterly Journal of Economics, 98, 85-106. https://doi.org/10.2307/1885568

[6] Mcdonald, R. and Siegel, D. (1985) Investment and the Valuation of Firms When There Is an Option To Shut Down. International Economic Review, 26, 331-349. https://doi.org/10.2307/2526587

[7] Pindyck, R.S. (1988) Irreversible Investment, Capacity Choice and the Value of the Firm. American Economic Review, 78, 969-985.

[8] Pindyck, R.S. (1991) Irreversibility, Uncertainty, and Investment. Journal of Economic Literature, 29, 1110-1148.

[9] Cobb, C.W. and Douglas, P.H. (1928) A Theory of Production. American Economic Review, 18, 139-165.

[10] Walras, L. (1874) Elements of Pure Economics. Harvard University Press, Cambridge, 1954.

[11] Keynes, J.M. (1936) The General Theory of Employment Interest and Money. Macmillan Co., Ltd., London.

[12] Ramsey, F.P. (1928) A Mathematical Theory of Saving. Economic Journal, 38, 543-559. https://doi.org/10.2307/2224098

[13] Solow, R.M. (1956) A Contribution to the Theory of Economic Growth. The Quarterly Journal of Economics, 70, 65-94. https://doi.org/10.2307/1884513

[14] Kydland, F.E. and Prescott, E.C. (1990) Business Cycle: Real Facts and a Monetary Myth. Reserve Bank of Minneapolis Quarterly Review, 14, 3-18.

[15] Kydland, F.E. and Prescott, E.C. (1991) The Econometrics of the General Equilibrium Approach to Business Cycle. Scandinavian Journal of Economics, 93, 161-178.

https://doi.org/10.2307/3440324 
Submit or recommend next manuscript to OALib Journal and we will provide best service for you:

- Publication frequency: Monthly

- 9 subject areas of science, technology and medicine

- Fair and rigorous peer-review system

- Fast publication process

- Article promotion in various social networking sites (LinkedIn, Facebook, Twitter, etc.)

- Maximum dissemination of your research work

Submit Your Paper Online: Click Here to Submit

Or Contact service@oalib.com 